\title{
Medication adherence and persistence in patients with autoimmune rheumatic diseases: a narrative review
}

This article was published in the following Dove Press journal:

Patient Preference and Adherence

\author{
Laura-Alexandra Anghel ${ }^{1, *}$ \\ Andreea Maria Farcaș ${ }^{2, *}$ \\ Radu Nicolae Oprean ${ }^{1, *}$ \\ 'Department of Analytical Chemistry \\ and Instrumental Analysis, Faculty \\ of Pharmacy, University of Medicine \\ and Pharmacy "Iuliu Hațieganu", Cluj- \\ Napoca, Romania; 'Drug Information \\ Research Centre, University of \\ Medicine and Pharmacy "Iuliu \\ Hațieganu”, Cluj-Napoca, Romania \\ *These authors contributed equally \\ to this work
}

Background: Several drugs are available for the treatment of autoimmune rheumatic diseases; however, their effectiveness may be negatively influenced by inappropriate adherence. Low adherence and persistence rates have a significant impact on patient quality of life and are associated with health-related expenses.

Purpose: To provide an up-to-date narrative review on treatment adherence and persistence rates, and discuss the factors that influence them, in patients with autoimmune rheumatic diseases.

Materials and methods: We searched the PubMed database for studies among patients with a diagnosis of rheumatoid arthritis (RA), ankylosing spondylitis (AS), systemic lupus erythematosus (SLE), or psoriatic arthritis (PsA), published from January 2015 to February 2017. Only studies with a well-defined measurement of adherence/persistence and those that carried out an evaluation of the influencing factors were included.

Results: Fifteen relevant studies that evaluated adherence and/or persistence were included. Adherence rates varied between $9.3 \%$ and $94 \%$, and persistence rates between $23 \%$ and $80 \%$. Most of the studies used one method to evaluate adherence or persistence (different questionnaire scores, proportion of days covered, and mean treatment duration). A high concordance was found between the adherence measurements of the Medication Event Monitoring System and Visual Analog Scale. Factors of economic, demographic, and clinical nature were only moderately linked to treatment adherence or persistence. However, patient-related factors - such as positive and increased beliefs in medication necessity, strong views of the chronic nature of the diseases, and increased knowledge of the disease - were related to better treatment adherence.

Conclusion: Owing to the heterogeneity of the study results, we consider that the use of more than one method to assess adherence/persistence should yield more comprehensive and accurate data about patient adherence behavior. Patient-related factors should be included and analyzed more often in adherence studies as the former may be modified to improve patient adherence. Keywords: drug therapy, rheumatology, patient nonadherence, risk factors

\section{Introduction}

As reported by the World Health Organization (WHO), patient adherence to long-term therapies is alarmingly low in both developed and developing countries. ${ }^{1}$ The impact of poor adherence on the effectiveness of chronic disease treatment is severe - both in terms of poorer health outcomes and increased health care costs. Low adherence impacts the quality of life of patients, affecting their ability to function in society. Furthermore, it increases the costs associated with the required medical interventions, rates of hospitalization, and increased visits to physicians. ${ }^{1-4}$ 
Studies in this area have validated the following statement: "Increasing the effectiveness of adherence interventions may have a far greater impact on the health of the population than any improvement in specific medical treatments" ${ }^{1,4}$

Medication adherence is a complex issue, and the different terminology used when analyzing this may cause debate and confusion. It is common to find studies that have the same measures referred to by different names: compliance, adherence, concordance, persistence, and discontinuation. These terms describe different aspects of patients' medication-taking behavior (extent of drug use, continuation of therapy, etc.) that are related to patients' knowledge and understanding of their treatment and disease, and also reflect the relationship with their health care professionals. Occasionally, some of these terms are used interchangeably; however, this is not entirely correct. Moreover, the use of multiple terms is even more confusing as most of these terms do not have a clear or direct translation into different European languages. ${ }^{1-7}$

As defined by the WHO, adherence represents "the extent to which a person's behavior - taking medication, following a diet, and/or executing lifestyle changes - corresponds with the agreed recommendations from a health care provider". ${ }^{1}$ In other words, adherence refers to "the extent of drug use during a period of persistence". ${ }^{2,4-7}$ In some cases, adherence and compliance are used as synonyms; in others, adherence is referred to as part of the compliance process.

Persistence is described as "the time of continuous therapy", referring to "the continuation of drug use for an overall duration of drug therapy". ${ }^{2-8}$ Depending on the source, persistence can be defined alternatively as the time between pharmacy refills or renewal of prescription (in most cases, allowing a gap of 30,45 , or 60 days). ${ }^{6-8}$

Parameters most often used to evaluate adherence and persistence are: medication possession ratio (MPR), proportion of days covered (PDC), survival time, retention rate, and different scores - depending on the method used for assessing them. ${ }^{2-11}$ There are both direct and indirect approaches to evaluate treatment adherence, each with advantages and disadvantages; however, ultimately, there is no single method that can accurately measure treatment adherence. ${ }^{2-18}$ Direct methods such as therapeutic drug monitoring and measurements of the drug or a metabolite provide a quantifiable value that offers evidence of drug ingestion. These are often referred to as the most "objective" and "direct" approaches to measure treatment adherence as they are subject to low bias; however, these approaches may be expensive and, sometimes, inconvenient for patients. Indirect methods such as pill count, electronic monitoring devices, electronic databases, and self-reported methods are most popular but can be subjective and overestimate adherence.

Autoimmune rheumatic diseases are a heterogeneous group of rare inflammatory conditions that share common immunopathogenic mechanisms. They are characterized by various clinical features and multiple organ involvement, and are associated with increased morbidity and mortality.

As in other chronic conditions, treatment adherence is an important part of their therapy. Because they involve lifetime treatments, the impact of low adherence is serious and can influence the effectiveness of the medication regimen. Unrecognized nonadherence could be wrongfully interpreted as an underestimation of treatment effectiveness.

International and national treatment guidelines exist: although they cover the management of these diseases, such guidelines offer no specific information or recommendations in regard to treatment adherence. ${ }^{19-22}$

Disease management for autoimmune rheumatic diseases consists of various pharmacological or non-pharmacological approaches. Diverse pharmacological options are available and include: corticosteroids, nonsteroidal anti-inflammatory drugs (NSAIDs), analgesics, and disease-modifying antirheumatic drugs (DMARDs). DMARDs comprise two major classes: conventional synthetic DMARDs (csDMARDs) and biological DMARDs (bDMARDs). ${ }^{19-22}$ Disease activity and clinical manifestations, comorbidities, and safety issues are some of the aspects taken into account when choosing an appropriate approach to offer patients the best possible quality of life and prevent inflammation and further structural damage. ${ }^{19-22}$ This can only be achieved if patients adhere to their treatments.

Demographic and economic aspects as well as therapy and disease-related factors, along with patient-related factors, are frequently assessed in adherence studies; however, to date, no predictors have been found to be strongly related to or to influence - nonadherent behavior. ${ }^{5,8-15}$ Furthermore, contradictory results have been reported. The inclusion of disease- (clinical factors, disease duration, and activity) and therapy-related factors (medication type, dosing frequency, previous treatments) in adherence studies focusing on autoimmune rheumatic diseases is based on existing knowledge of their relationship with adherence in other chronic diseases. ${ }^{1}$ Adherence is simultaneously influenced by several factors; some of these are potentially modifiable, with potential for use in screening to identify nonadherent patients. These factors demonstrate the importance of accurate identification of the various reasons for patient nonadherence to treatment plans. 
Nonadherence is commonly categorized into two groups: unintentional - which can be related to inaccessibility to medication, language barriers, polypharmacy, and forgetfulness - and intentional, which is strongly related to patients' personal beliefs, decisions, and treatment. ${ }^{4,8-16,23}$

This study was conducted to offer an up-to-date overview of the existing information available on rates of adherence and persistence in patients affected by autoimmune rheumatic diseases, and to include factors that potentially influence these rates. An accurate view on this subject would contribute to increased knowledge and improve the effectiveness of therapies. We included studies that evaluated either adherence or persistence because, in essence, both are distinct aspects that relate to the same topic.

\section{Materials and methods}

We conducted a literature search to identify studies on patient adherence to their treatments and the factors that potentially influence it.

\section{Search strategy}

A PubMed search was conducted with the start date January 1, 2015, and end date February 20, 2017. This interval was chosen on the basis of relevance; only the latest studies were included as reviews including older studies are already available.

\section{Terms used in the search}

The terms "persistence" or "adherence" or "compliance" or "discontinuation" AND "rheumatoid arthritis" or "ankylosing spondylitis" or "systemic lupus erythematosus" or "psoriatic arthritis" AND "treatment" or "therapy" or "medication" were searched.

Only English-language articles and those conducted on adults ( $>18$ years) were included.

Reviews, case reports, letters, and editorials were not included as primary data in this review. Each article was screened and assessed for relevance of results on adherence by reading the abstracts or the full text.

\section{Findings based on search criteria}

Briefly: 186 articles on rheumatoid arthritis (RA) were selected, of which 28 articles were considered potentially relevant; 35 articles on systemic lupus erythematosus (SLE) were identified, of which 11 were considered potentially relevant; 23 articles on ankylosing spondylitis (AS) were found, of which six were considered potentially relevant (after eliminating duplicates, only two remained); and 26 articles on psoriatic arthritis (PsA) were short-listed, of which five were considered potentially relevant (after eliminating duplicates, two remained).

Full-text articles were retrieved for the remaining 43 articles and, in the present narrative review, we included only those articles that met the following inclusion criteria:

- Studies containing a well-defined measurement of adherence/persistence and reporting adherence/ persistence as an outcome.

- Studies reporting an analysis of associated, predictive, or risk factors related to adherence.

Following these criteria, 15 studies were included in the present narrative review.

\section{Results}

Adherence, as an outcome, was assessed in ten out of 15 , persistence in two, and drug discontinuation in three studies. One study evaluated both adherence and treatment abandonment, ${ }^{24}$ and two studies reported results for both adherence and persistence rates. ${ }^{25,26}$ The sample size in the studies ranged from 80 to 12,893 participants. Participants were derived either from the outpatient clinic $^{27-32}$ or were recruited online ${ }^{33}$ through social media or forums, or were patients from established cohorts in medical databases. ${ }^{24-26,34,35}$ In two studies, the Danish nationwide DANBIO Registry, which includes clinical data on patients with rheumatic diseases treated with biologics in routine care, was used. ${ }^{36,37}$ Another study recruited patients through the British Society for Rheumatology Biologics Register for RA - a UK-wide prospective observational cohort study established in 2001 for the purpose of monitoring the long-term safety of biologic therapy.$^{38}$ In regard to study design, four had a cross-sectional design, ${ }^{27,28,31,32}$ five were retrospective cohort studies, ${ }^{24-26,34,35}$ and six were prospective studies..$^{29,30,33,36-38}$

\section{Adherence and persistence rates and measurements}

There was considerable variation in regard to the terms and concepts related to adherence and persistence between studies. Different definitions were used, as presented in Table 1.

The majority of the studies estimated adherence for RA patients, ${ }^{24-31,33-35,38}$ and some included both RA and AS patients. ${ }^{31,33-35}$ PsA patients were included in three studies, ${ }^{33,34,37}$ and one study included patients with SLE. ${ }^{32}$

Most of the studies applied a single method to evaluate adherence, whereas only two studies used more than one method. ${ }^{28,29}$ Self-reported adherence was the most 


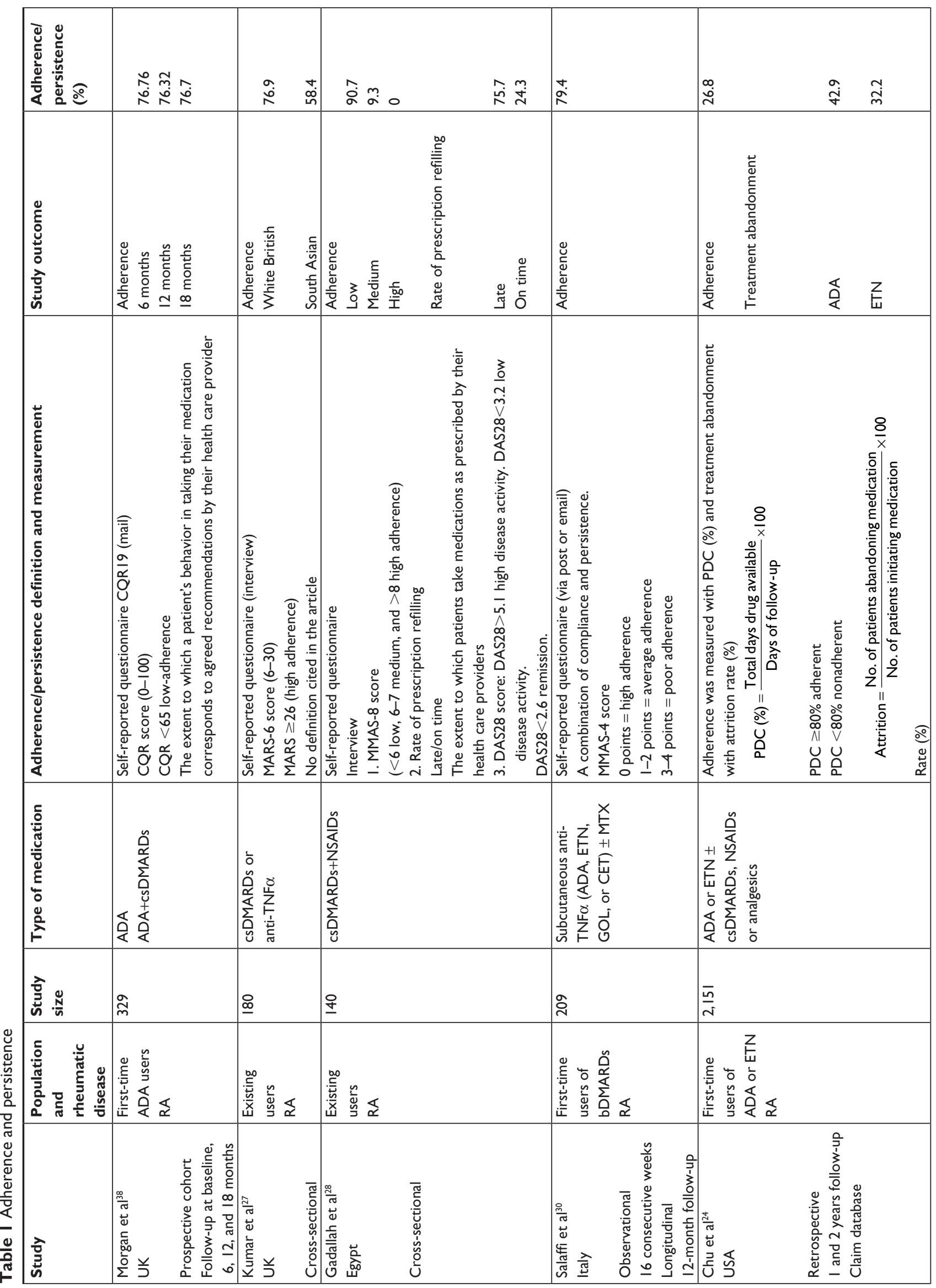




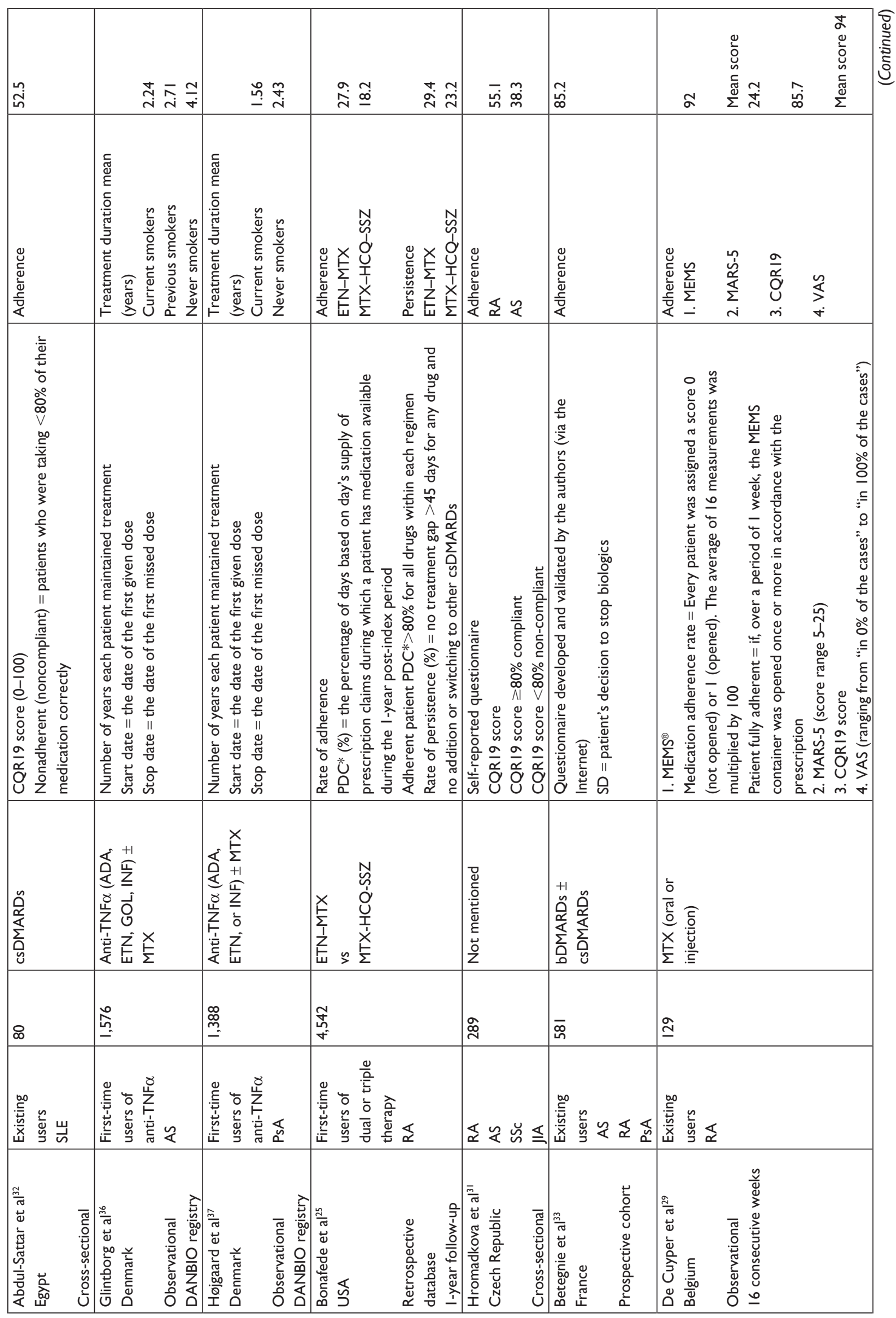




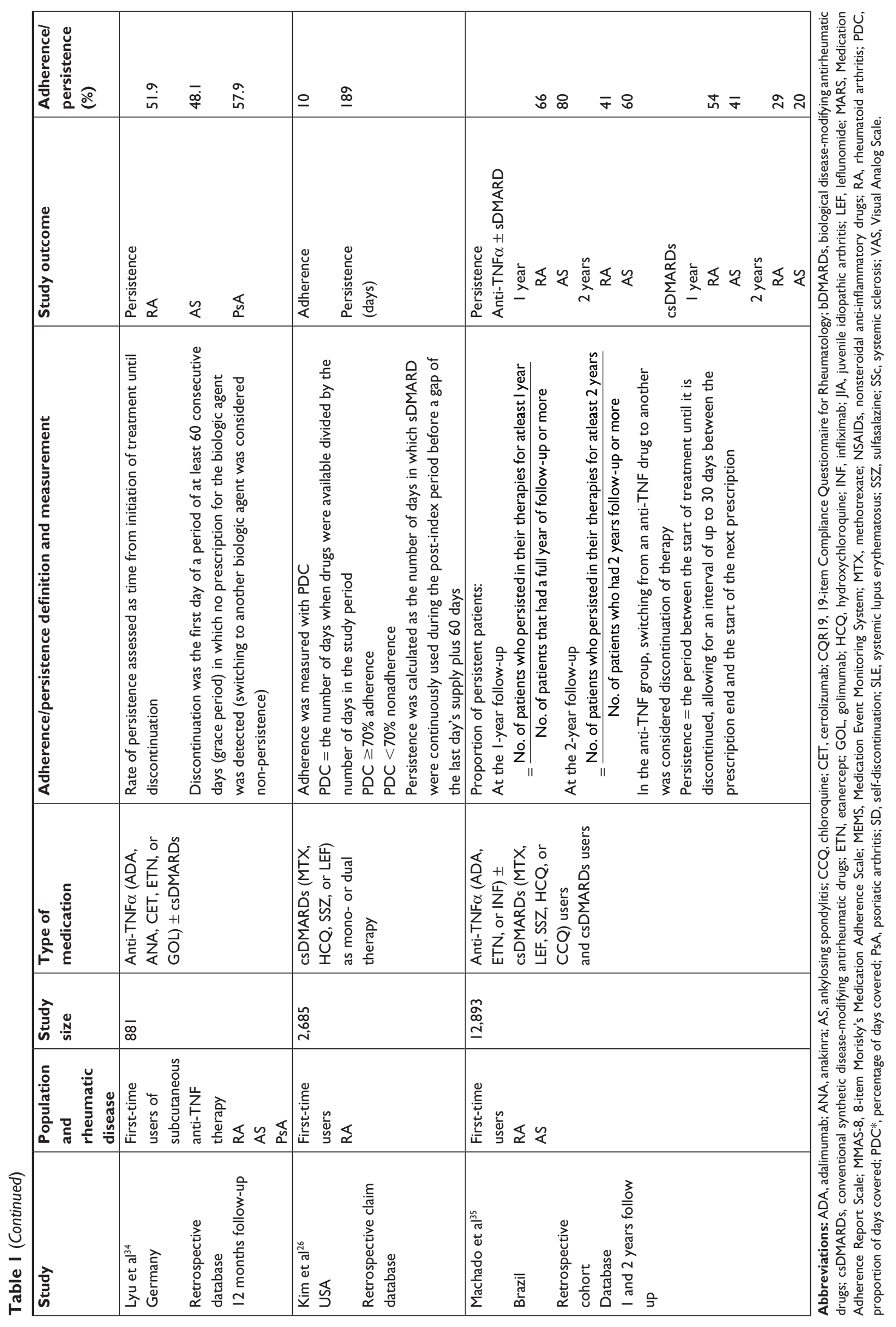


frequently used method, with different questionnaires being employed - the 19-item Compliance Questionnaire for Rheumatology-19 (CQR19), which was created specifically for and validated to use in rheumatic diseases, ${ }^{29,31,32,38}$ eight- and 4-item Morisky's Medication Adherence Scale (MMAS-8 and MMAS-4, respectively), and 6- and 5-item Medication Adherence Report Scale (MARS-6 and MARS-5, respectively), ${ }^{27-30}$ in one of the studies, the investigators developed and validated a special questionnaire. ${ }^{33}$

When measuring adherence using medical databases, PDC was used in three of the studies, ${ }^{24-26}$ and rates of persistence were the outcome in four others. ${ }^{25,26,34,35}$ One study gave information on treatment abandonment, which was assessed with the attrition rate. ${ }^{24}$ More details on the methods of calculation for all these studies are presented in Table 1.

Some of the studies included rates of adherence in existing users of medication; ${ }^{27-29,32,33}$ however, the majority assessed adherence or persistence for first-time users, ${ }^{24-26,30,34-38}$ whereas one study did not mention this aspect. ${ }^{31}$ Most commonly, first-time users referred to patients initiating biologic therapy.

Rates of adherence varied widely between $9.3 \%$ and $94 \%$, with results depending on the rheumatic disease, the method used to assess adherence, as well as the cutoff point that was used to separate nonadherent from adherent patients. The lowest adherence was detected in a cross-sectional study, with $9.3 \%$ of the RA patients being classified as mediumadherent according to the MMAS- 8 measurement. ${ }^{28}$ None of the patients included met the criteria for being high adherers. The highest rate of adherence was measured in an RA cohort receiving methotrexate (MTX). ${ }^{29}$ The results obtained using the Medication Event Monitoring System (MEMS) method ( $92 \%$ of patients adhered to treatment) correlated the highest with the results from the Visual Analog Scale (VAS) mean score of self-reported adherence (94\%). ${ }^{29}$

Rates of persistence varied widely across studies, ranging between $23 \%$ and $80 \%$. A low persistence was found in RA patients treated with MTX-HCQ-SSZ (methotrexatehydroxychloroquine-sulfasalazine) triple therapy (23.2\%). A high rate of persistence was found in AS patients undergoing anti-tumor necrosis factor alpha (anti-TNF $\alpha$ ) therapy with or without concomitant csDMARD use $-80 \%$ in the first year of follow-up, decreasing to $60 \%$ in the second year.

\section{Factors associated with adherence}

A variety of associated/predictive factors were analyzed in all the studies, including sociodemographic and economic factors, therapy- and disease-related factors, and patient-related factors; however, only a small number of these factors was found to influence adherence or persistence.

\section{Social and economic factors}

Sociodemographic factors, such as age, ethnicity, gender, marital status, educational level, living situation, and employment status, were among those most commonly included in the analyses.

Results show that older patients with RA were more likely to be adherent, ${ }^{24,30,38}$ whereas another study found that younger patients with RA were more likely to adhere to their therapies. ${ }^{28}$ No other study reported age as a predictor of patient adherence behavior.

For SLE patients, factors such as very low and low economic status, lower education levels, and rural residency were found to be correlated with adherence in a negative way. ${ }^{32}$ Another study detected that RA patients who had a lower income were more likely to be persistent in the first and second year of follow-up than those with better incomes. ${ }^{35}$

The connection between smoking status and treatment adherence was evaluated in two studies from Denmark using data from the DANBIO registry. ${ }^{36,37}$ One of them found that AS patients who were current and previous smokers had poorer treatment adherence than never smokers, with this finding being relevant mainly in men. ${ }^{36}$ These results were consistent regardless of the TNF- $\alpha$ inhibitor prescribed. When they compared previous smokers with never smokers, the authors found that previous smokers had poorer adherence for adalimumab (ADA) and etanercept (ETN) ${ }^{36}$ The same registry was used to assess the influence of smoking status on treatment adherence in PsA patients, and current smoking status was associated with poorer adherence to ETN and infliximab (INF), but not to ADA. ${ }^{37}$

Increased professional or familial support was associated with greater adherence, ${ }^{33,38}$ whereas living alone had a negative impact on adherence. ${ }^{29}$ Two out of three studies that included the patients' ethnicity found a relevant connection with treatment adherence. ${ }^{24,27}$ White British patients with RA had better treatment adherence than South Asians, ${ }^{27}$ and African-American patients with RA were more likely not to adhere to their first bDMARD. ${ }^{24}$ Details of these factors from all studies are presented in Table 2.

\section{Health system-related factors}

Health system-related factors were evaluated in more than half of the studies, ${ }^{24,25,27,28,30,33,34,38}$ referring to either the type of insurance (in studies conducted in databases) or the different aspects relating to physician interaction (language used in 


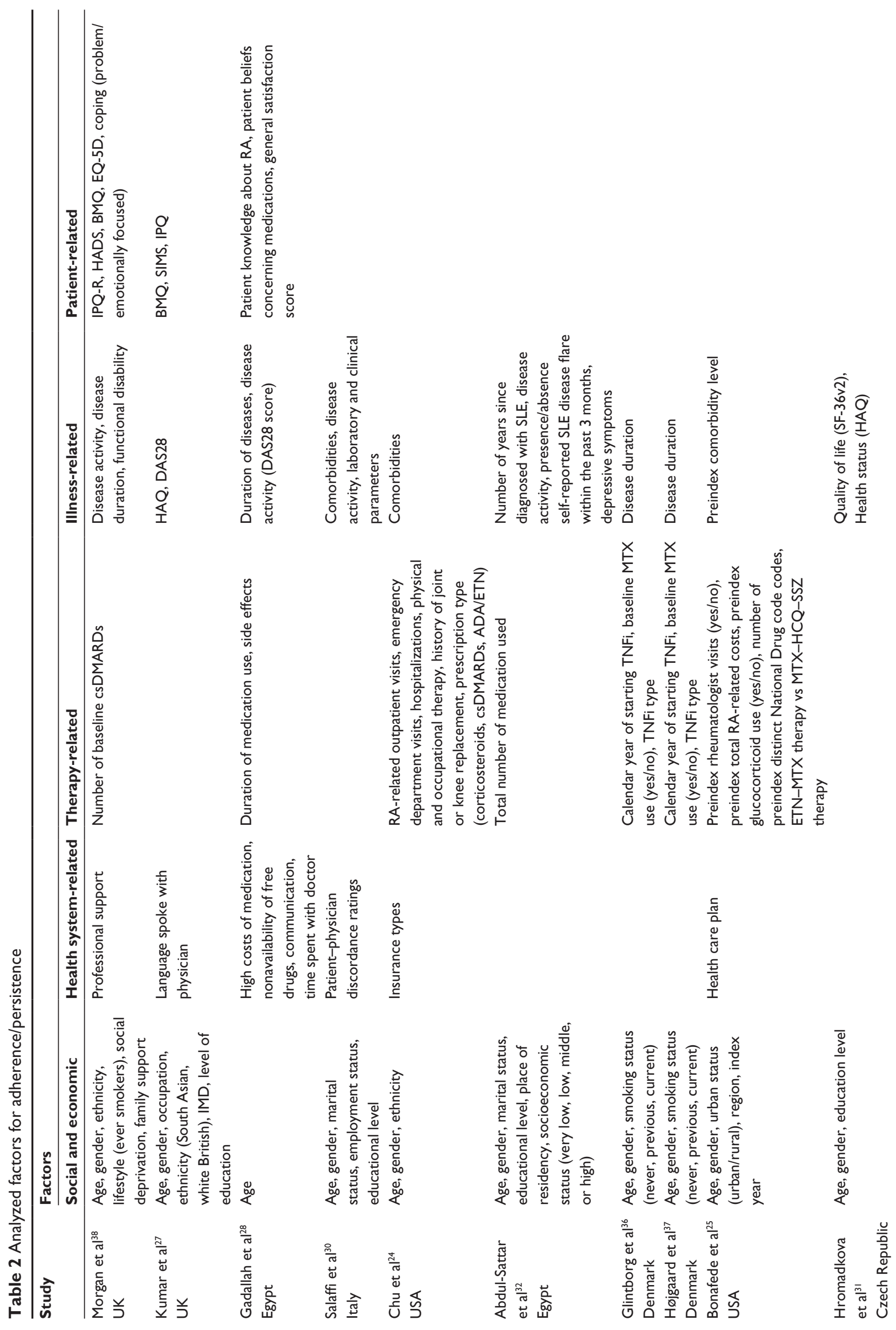



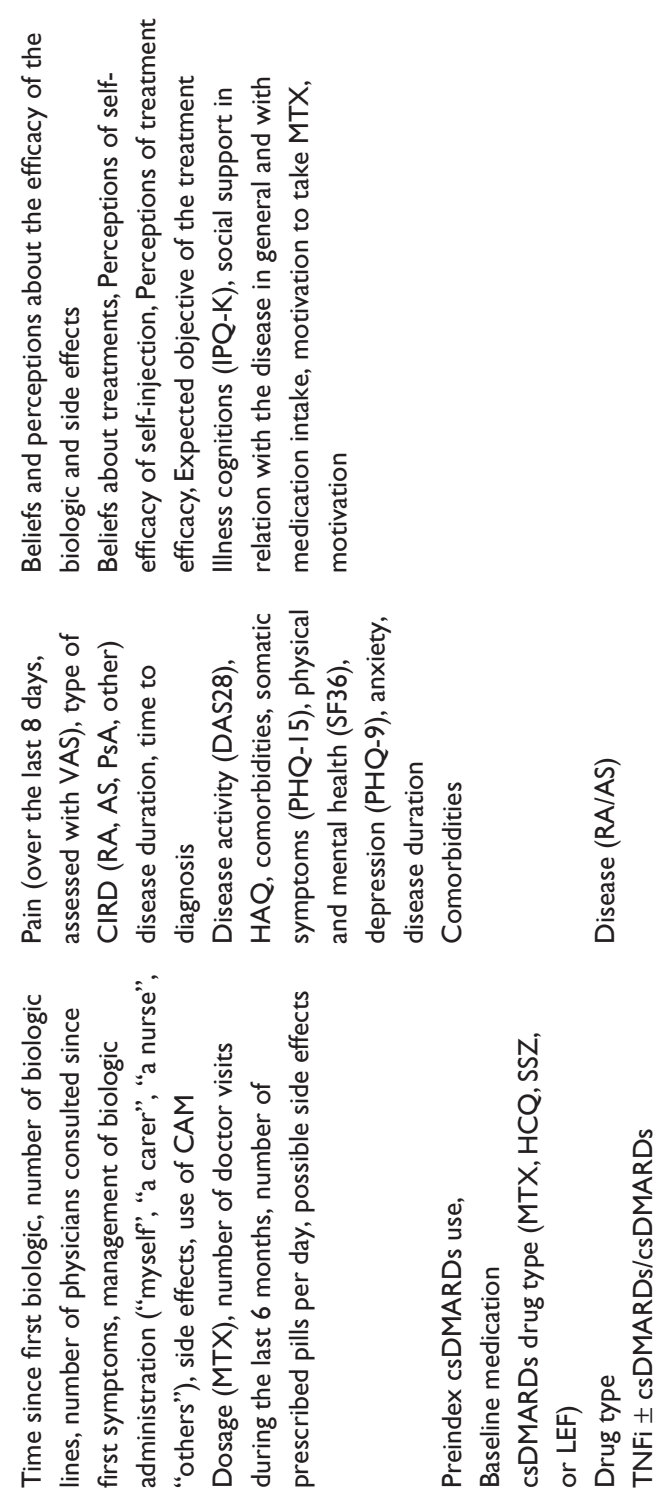

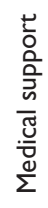
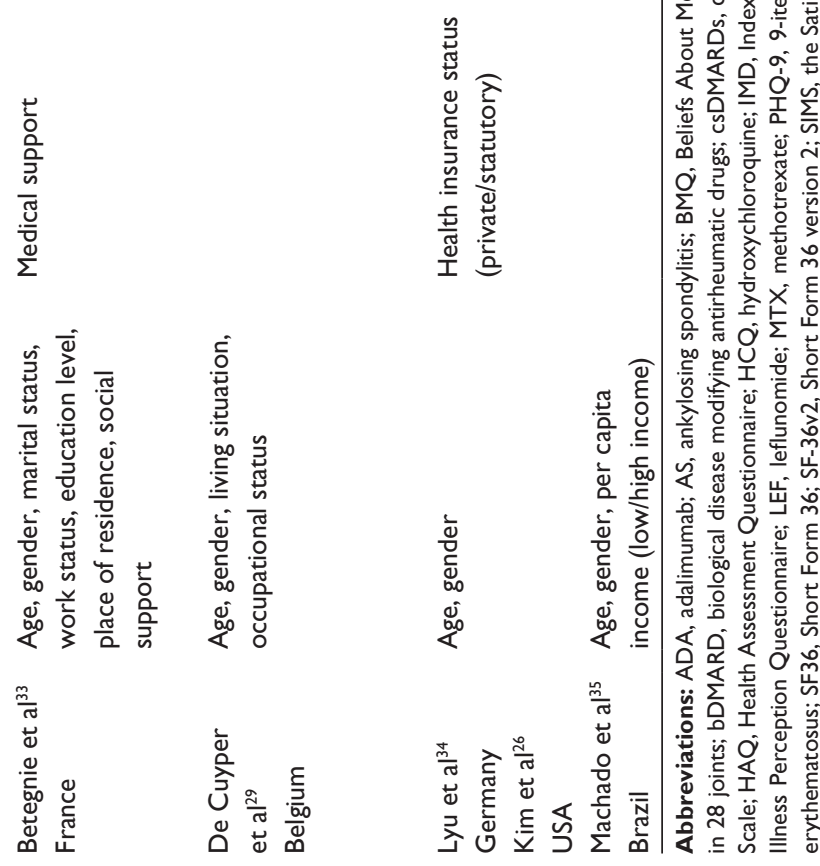

communication and professional support with discordance rates). The findings were not conclusive, with just three studies reporting a significant correlation between health system-related factors and adherence. ${ }^{28,30,33}$

Nonavailability of cost-free drugs in the pharmacy is, as expected, one of the barriers to treatment adherence. ${ }^{28}$ Lack of perceived medical support ${ }^{33}$ and higher patient-physician discordance rates ${ }^{30}$ had a negative impact on treatment adherence.

\section{Therapy-related factors}

Different factors related to therapy, such as type of medication used, complexity of the treatment regimen, side effects, and duration of medication used were included in 12 of the 15 studies and found to have a relevant connection to adherence or persistence in some of them, ${ }^{24-26,32-35}$ being mostly related to the type of medication used.

Factors found to be positively associated with both adherence and persistence were csDMARD monotherapy (with either MTX or LEF) ${ }^{26}$ and ETN-MTX use in RA patients. ${ }^{25}$ Factors positively influencing persistence were existing csDMARD RA users ${ }^{34}$ and anti-TNF $\alpha$ therapy with or without csDMARDs in AS patients. ${ }^{35}$

ETN use in RA patients and an increased number of medications used by SLE patients were found to have a negative impact on adherence. ${ }^{24,32}$ More than one attempted and selfadministered bDMARD therapy was also a factor that had a negative impact on self-discontinuation, which was defined as the patient's own decision to stop the treatment "alone" or "alone and then validated by a physician". ${ }^{33}$

\section{IIIness-related factors}

A wide range of illness-related factors, such as type of disease and disease duration, disease activity and functional disability, depressive symptoms, and other comorbidities, was included in most studies. Most of the reported results were inconsistent, making it difficult to establish a coherent pattern.

Longer disease duration, ${ }^{28,38}$ lower levels of pain, ${ }^{33}$ and both low levels ${ }^{30}$ and high levels ${ }^{28}$ of disease activity were found to have a negative impact on adherence. Better mental health status predicted better adherence..$^{29,32}$

The presence of comorbidities (coronary artery disease, hypertension, COPD, renal disease, and liver disease) was found to have both a negative ${ }^{24,30}$ and a positive impact on treatment adherence. ${ }^{29}$

\section{Patient-related factors}

The patient's knowledge about their disease, motivation to take medicines, and the patient's perceptions about efficacy 
and concerns about therapy or side effects are some of the related factors included in the studies. ${ }^{27-29,33,38}$ Beliefs and perceptions about treatments were evaluated using the Beliefs About Medicines Questionnaire (BMQ) 27,38 or other scales. ${ }^{28,33}$ Positive and increased beliefs in medication necessity were associated with higher rates of adherence, ${ }^{27,28,33,38}$ and lower medication concerns had a positive effect on adherence. ${ }^{27,38}$ Strong views of the chronic nature of the diseases ${ }^{38}$ increased knowledge of the disease, ${ }^{28}$ satisfaction with information received about therapy, ${ }^{27}$ and greater satisfaction score ${ }^{28}$ were all factors associated with greater treatment adherence.

A simplified list of all the factors enclosed, and the direction of association with adherence and persistence, is presented in Table 3.

\section{Discussion}

\section{Adherence and persistence rates and measurements}

Patients who adhere to their treatments are three times more likely to achieve desired outcomes, such as improved quality of life and better functional capacity, than nonadherent patients. ${ }^{39}$ However, research suggests that adherence rates drastically drop after 6 months of treatment; this is valid in a number of chronic diseases such as cardiovascular conditions and hypertension, asthma, diabetes, and RA. ${ }^{1,40}$ Chronic patients might display a number of common adherence characteristics, some being closely related to the specific features of the disease that they suffer from, with each facing unique and distinctive challenges.

We found that rates of adherence vary widely in the four autoimmune rheumatic diseases included in this review, underlining the seriousness and complexity of this aspect. In previous reviews of earlier studies, there are the same wide variations, with reported adherence rates in rheumatic diseases ranging between $7 \%$ and $75 \%{ }^{4}$

The diversity of the definitions and methods used to evaluate adherence and persistence might explain the variation in results. There is no standard method to evaluate adherence, and the choice remains entirely at the hands of the investigators conducting the study, and varies based on the resources, desired outcome, and personal interpretations on the matter. However, the different methods used in the studies from this review assessed various aspects of treatment adherence. The findings should, therefore, not be discarded, but rather, analyzed and integrated in the wider context as part of understanding the complex patient-treatment behavior. As there is no "gold standard" for evaluating adherence, using two methods (eg, MEMS and a self-reported method) may lead to more accurate measurement of patients' treatment adherence, as they gather sets of information by using different approaches and perspectives, thereby complementing each other. Using both a subjective and an objective method could also provide additional information on the beliefs and barriers pertaining to adherence. ${ }^{12}$ In the study using four methods for evaluating adherence in patients taking MTX, the highest concordance was found between MEMS, an objective method, and VAS, a subjective method - with the latter being frequently perceived to overestimate adherence. ${ }^{29}$ However, this study demonstrated that VAS may be used in daily practice as a quick and simple method for screening medication adherence.

Adherence is a dynamic process that changes over time; therefore, a complex image can only be obtained if adherence is evaluated both at the beginning of a treatment and during the continuation phase. This could partly explain the diversity of adherence rates in the studies included here, as some of them measured adherence in patients initiating a new treatment regimen (most frequently, the initiation of an anti-TNF $\alpha$ agent) and some evaluated adherence in existing users. Longitudinal studies - commencing at the start of a treatment and following patients through the years of treatment - could give a complete representation of adherence and inform physicians about the different factors influencing it along the way.

Data on direct comparisons between rates of adherence and persistence between different diseases were available for RA and AS patients. Although it is difficult to draw a clear conclusion, RA patients tended to have slightly higher rates of adherence than AS patients. ${ }^{31,33,34}$

In three of the studies, patients responded to adherence questionnaires online, showing overall better adherence..$^{30,33,38}$ The selection of recruitment strategy could bias the results, by choosing some categories of patients (younger, better education, and better social status) and excluding others. Moreover, it could lead to results that reflect reality better, with patients that do not display "white coat adherence behavior".

\section{Factors associated with nonadherence}

According to the WHO, there are five dimensions of factors influencing medication adherence: social and economic factors, health system-related factors, therapy-related factors, illness-related factors, and patient-related factors. ${ }^{1}$

A broad range of social and economic aspects that characterize the personal context of the patient have been included in almost all of the studies. These aspects are quite easy to 


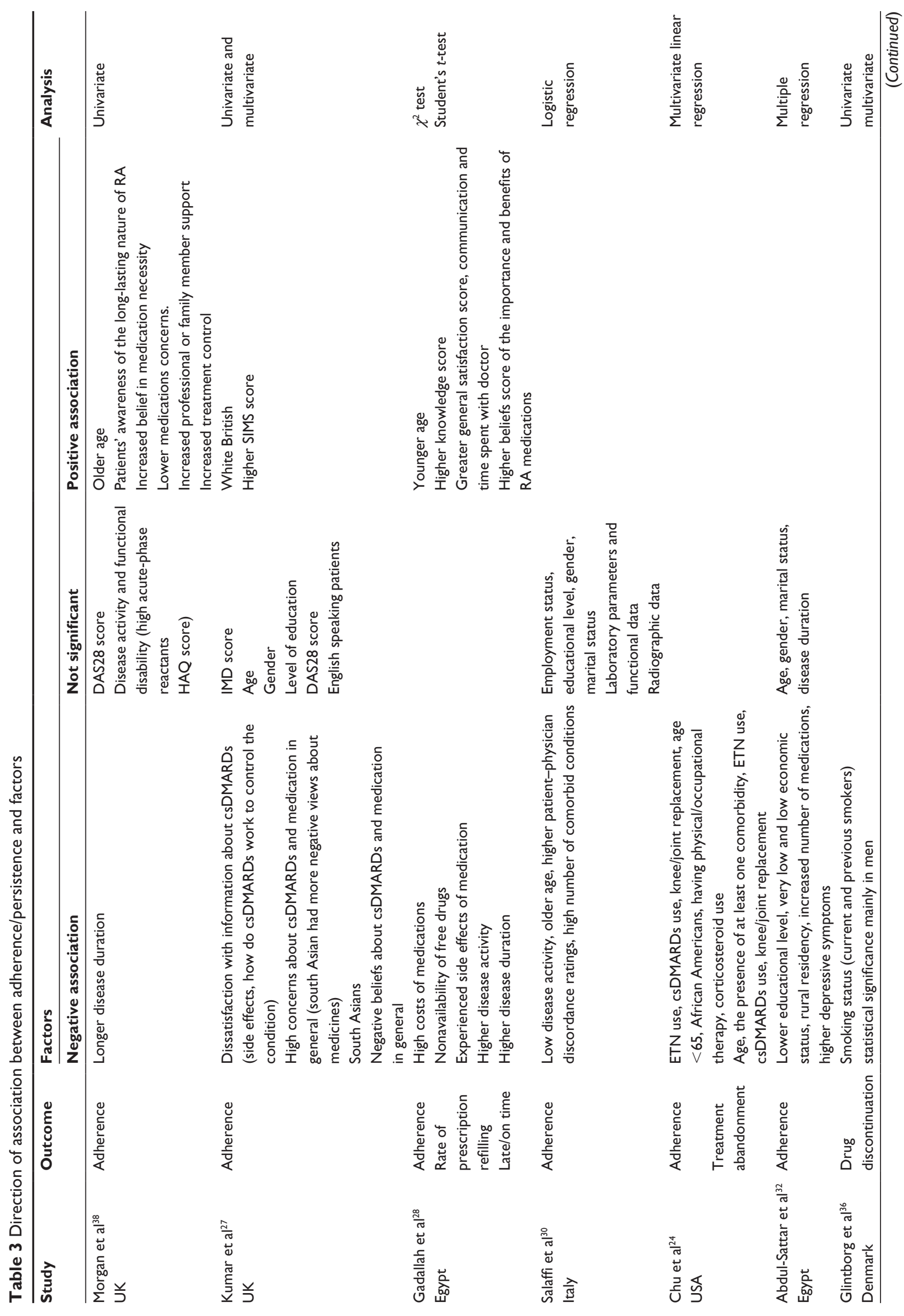




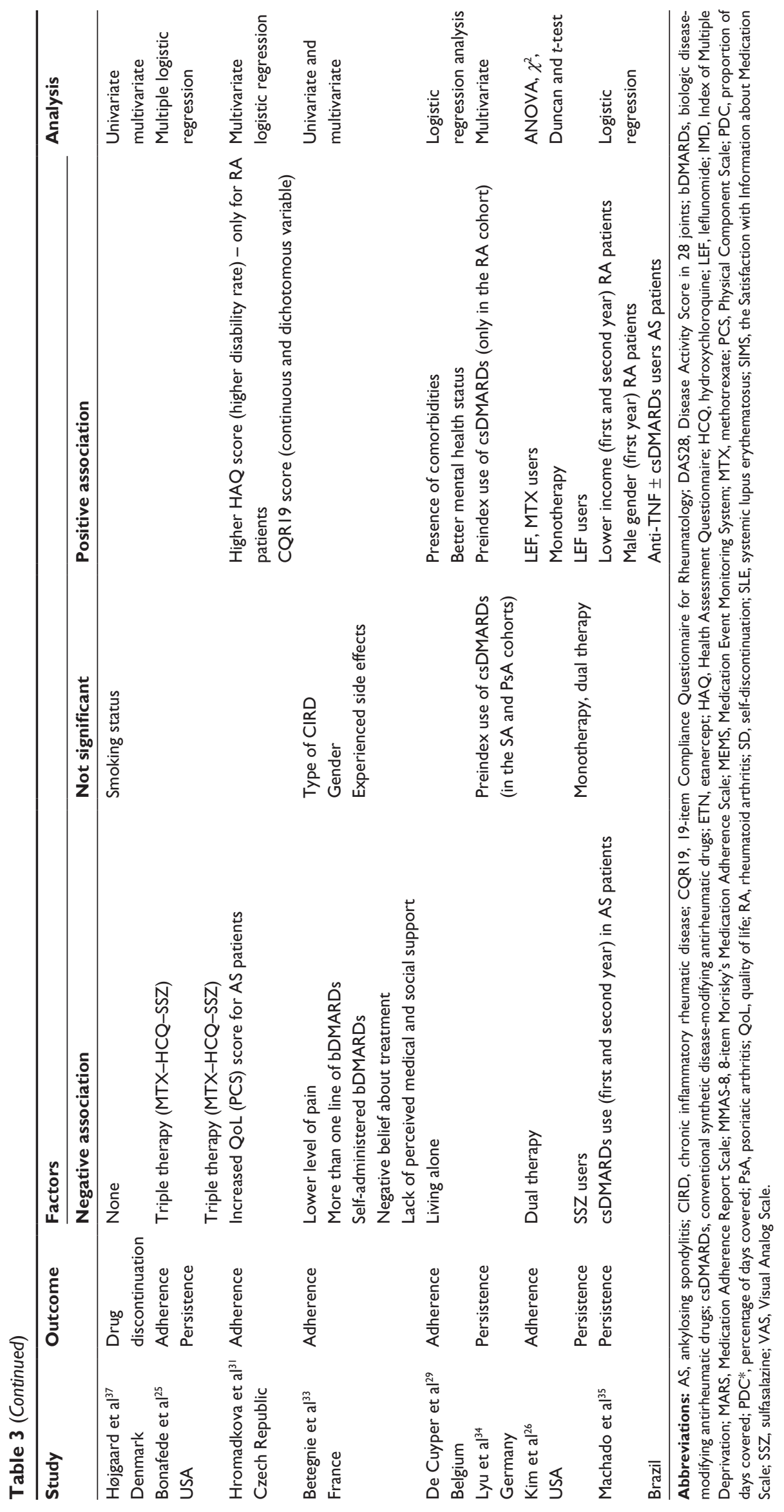


obtain, regardless of the method used to evaluate adherence or persistence. There are, however, no consistent theories that explain why these factors should be included and what is the extent of their influence on adherence. Moreover, they may have limited value due to the fact that they are not modifiable. However, they could be considered for risk screening and targeted interventions. ${ }^{10}$ Altogether, they have been associated with treatment adherence in diabetes, epilepsy, HIV, and statin use, but the association with rheumatic diseases is still unclear. ${ }^{1,10}$

The most studied aspect - the influence of age on adherence - was found to be relevant in few of the studies we analyzed and showed opposite results, consistent with similar findings from other reviews and studies. ${ }^{8,10,11,13,14,40}$ We did not find an association between gender and treatment adherence, but there is evidence in literature that links female gender to increased risk of biologic discontinuation..$^{8,40}$ One factor in particular - social support (from family and community) - was shown to have a positive impact on adherence, ${ }^{33,38}$ whereas living situation (living alone) had a negative impact on adherence. ${ }^{29}$ This is valid for other diseases and shows the importance of maintaining an optimal level of interaction and support that patients need in order to adhere to their treatments. ${ }^{1,41}$ In a few studies, smoking status has been linked to the effectiveness of treatment in patients with RA and PsA, making it an important factor to be included in adherence research, as it is also potentially modifiable. ${ }^{42,43}$ These findings are in line with the ones from two studies in our review. ${ }^{36,37}$ Ethnicity, which was found to influence adherence in RA patients, ${ }^{24,27}$ does not appear to be a consistent predictor of adherence in some reviews, ${ }^{4,10,11}$ whereas it seems to influence adherence in others. ${ }^{9,44}$ A strong connection between other social and economic factors has not been established in other studies either. ${ }^{4,8,10,11,13}$

Findings from our review suggests that some of the health system-related factors (eg, patient-physician relationship) contribute to treatment adherence. ${ }^{30,33}$ Other studies in this area suggest the same association, that a good relationship with the treating physician improves adherence outcomes, both in rheumatic diseases ${ }^{9,11,13,15,45}$ and in chronic conditions. ${ }^{1,41}$ This might actually explain the association between adherence and some patient-related factors. Patients likely have an increased trust in the treatment efficacy and stronger treatment beliefs if they feel they can rely on and trust the treating physician. Moreover, international guidelines promote patient implication in the prescription process as a ground principle of therapy. ${ }^{19-22}$ The trust RA patients had in their physicians was, in fact, shown to be one of the most important contributing factors when starting and adhering to an sDMARD treatment. ${ }^{46}$ This supports the concept that adherence is not just an individual characteristic, but rather, a complex and dynamic experience in which each part - patient, health care practitioner, and the community plays a specific role.

As patients with rheumatic diseases use complex treatment regimens, therapy-related factors were also assessed in the majority of the studies analyzed in this review. We have found that patients taking fewer medicines were more likely to be adherent than patients taking more medicines. ${ }^{25,26,32}$ Polypharmacy is widely recognized to raise safety concerns and influence adherence to treatment in a number of chronic conditions, ${ }^{1,47-49}$ including some rheumatic diseases, ${ }^{50}$ although this association was not always consistent among studies conducted on RA patients. ${ }^{10,11,13}$ The heterogeneity of these findings might be attributable to the diverse treatment regimens that are usually prescribed for these patients, which makes a direct and conclusive comparison difficult. Thus, adherence to MTX was better when compared to other csDMARDs, ${ }^{10,26}$ but not superior to bDMARDs. ${ }^{14,44}$ Among bDMARDs, there are studies that support a better adherence to subcutaneous ETN measured in lower discontinuation rates $^{4,8,40}$ than the adherence to intravenous INF (probably due to the implication of another health care provider, as INF is administered intravenously). Better adherence to ETN might also be explained by the low level of non-immunogenicity, compared to ADA and INF. ${ }^{51}$ Furthermore, we have found lower persistence rates for INF when compared with other anti-TNF $\alpha$ agents used in RA and AS patients. ${ }^{35}$

Factors related to the disease, have been extensively studied in relation with medication adherence in a wide range of chronic disorders. Laboratory parameters that assess the severity of the diseases are routinely measured at doctor visits and can potentially be used for adherence screening, if found related to adherence. The relationship between adherence and disease severity can be bidirectional. Disease severity could be both the cause and effect of adherence, especially in rheumatic diseases where manifestations include symptoms such as severe pain, stiffness, and multi-organ involvement. Until now, a relationship between adherence and disease duration or disease severity has been established in diabetes, hypertension, and epilepsy, ${ }^{1}$ but the findings are still inconsistent in autoimmune diseases., ${ }^{40,11,13,14,52}$ Moreover, we have found conflicting results among the studies screened in this review. It is difficult to state if the results are because of the actual lack of correlation or other confounders that might have influenced the results, such as medication type, 
follow-up period, and method of adherence measurement that cannot grasp the association. However, it is known that poor adherence leads to increased disease activity. ${ }^{53}$ Better mental status is associated with better adherence - both in our findings $\mathrm{s}^{29,32}$ and in previous reviews. ${ }^{4,9}$

The last category of factors related to medication adherence are those considered to be patient-related - that means factors connected to the patients' attitudes, perceptions, beliefs, and lifestyle habits. They can indirectly influence some of the other factors. People's perceptions of their medications can be divided with respect to beliefs about the necessity of taking the medication and concerns about taking it. 4,16,54,55 These have been found to be consistent predictors of adherence in a number of disorders, namely asthma, renal disorders, cancer, diabetes, mental illness, and coronary heart disease, as well as in immune-mediated inflammatory diseases. . $^{1,4-11,13,15,44,54-59}$ In some diseases, addressing the patients concerns seems more important than pointing out the necessity of treatment, ${ }^{57,58}$ whereas, in rheumatic diseases, convincing patients of the treatment's necessity seems more relevant. ${ }^{13,44,56}$ Similar consistent associations between adherence and increased necessity beliefs were observed by other groups. ${ }^{27,28,33,38}$

\section{Limitations}

Our results may have been influenced by a number of factors: 1) the heterogeneity of the studies included and inequality of the patient population covered (most studies involved RA patients, with the other rheumatic diseases thus being poorly represented); 2) methodological differences might have led to different adherence results (different methods used for assessment, some more "stricter" than others, that could have contributed to the ample variations of the results); and 3 ) potential confounders or specific elements could have influenced the results.

The ample variations of rates of adherence and persistence resemble the findings from systematic reviews, suggesting that our study - although not representing a systematic review - covers a relevant selection of the literature. Moreover, the results of our cumulative review present the latest findings in adherence research as we included studies published from 2015 to 2017 . These studies include therapeutic regimens that are in line with the most recent international treatment recommendations and guidelines, making the present review one of current interest.

From the large number of factors included in all of the studies, only a few were found to have a certain influence on adherence or persistence. This lack of association may be the result of the true absence of a relationship or could be caused by the heterogeneity of the studies. Although studies have shown similar efficacy in RA when compared to TNF $\alpha$ inhibitors, T-cell co-stimulation inhibitors (eg, abatacept) and interleukin (IL)-6 antagonists (eg, tocilizumab) are much less used in clinical practice. None of the studies included in our review had patients treated with either abatacept or tocilizumab; therefore, unfortunately, we could not provide data on treatment adherence or persistence in regard to these agents. One study did include patients with an IL-1 inhibitor (anakinra) but did not report adherence results to it, because the number of patients taking it was too small. ${ }^{34}$ The cross-sectional nature of four of the studies makes it challenging to establish a causal relationship between the findings, this being an issue noted by a significant number of systematic reviews. The retrospective database studies could only investigate the factors that were included in the databases; other factors that could have been potentially relevant, therefore, remain unexplored. Prospective data collection may represent a better choice; this was undertaken in only six of the 15 studies included in the present review.

\section{Conclusion}

Estimates of treatment adherence and persistence were shown to vary considerably because of differences in patient populations, follow-up durations, different types of adherence definitions, and measurements used.

Factors that suggest a coherent connection with adherence, such as personal beliefs and concerns, should more often be included in adherence research as there is some evidence to sustain their importance. Further research should focus on characterizing the specific relationship between treatment adherence and these factors. Future efforts should additionally aim to develop methods to improve treatment adherence in patients with autoimmune rheumatic diseases, thereby improving treatment effectiveness and patient quality of life.

\section{Disclosure}

The authors report no conflicts of interest in this work.

\section{References}

1. W H O. Adherence to long-term therapies: evidence for action; 2003. Available from: http://www.who.int/chp/knowledge/publications/adherence_report/en/. Accessed June 23, 2017.

2. van Mierlo T, Fournier R, Ingham M. Targeting medication nonadherence behavior in selected autoimmune diseases: a systematic approach to digital health program development. PLoS One. 2015;10(6): e0129364. 
3. De Vera MA, Mailman J, Galo JS. Economics of non-adherence to biologic therapies in rheumatoid arthritis. Curr Rheumatol Rep. 2014; 16(11):460.

4. Vangeli E, Bakhshi S, Baker A, et al. A systematic review of factors associated with non-adherence to treatment for immune-mediated inflammatory diseases. Adv Ther. 2015;32(11):983-1028.

5. Wong PK. Medication adherence in patients with rheumatoid arthritis: why do patients not take what we prescribe? Rheumatol Int. 2016;36(11):1535-1542.

6. Cramer JA, Roy A, Burrell A, et al. Medication compliance and persistence: terminology and definitions. Value Heal. 2008;11(1):44-47.

7. Vrijens B, De Geest S, Hughes DA, et al. A new taxonomy for describing and defining adherence to medications. $\mathrm{Br} J$ Clin Pharmacol. 2012;73(5):691-705.

8. López-González R, León L, Loza E, Redondo M, Garcia de Yébenes MJ, Carmona L. Adherence to biologic therapies and associated factors in rheumatoid arthritis, spondyloarthritis and psoriatic arthritis: a systematic literature review. Clin Exp Rheumatol. 2015;33(4):559-569.

9. de Achaval S, Suarez-Almazor ME. Treatment adherence to diseasemodifying antirheumatic drugs in patients with rheumatoid arthritis and systemic lupus erythematosus. Int J Clin Rheumatol. 2010;5(3): 313-326.

10. Scheiman-Elazary A, Duan L, Shourt C, et al. The rate of adherence to antiarthritis medications and associated factors among patients with rheumatoid arthritis: a systematic literature review and metaanalysis. J Rheumatol. 2016;43(3):512-523.

11. van den Bemt BJ, Zwikker HE, van den Ende CH. Medication adherence in patients with rheumatoid arthritis: a critical appraisal of the existing literature. Expert Rev Clin Immunol. 2012;8(4):337-351.

12. Shi L, Liu J, Fonseca V, Walker P, Kalsekar A, Pawaskar M. Correlation between adherence rates measured by MEMS and selfreported questionnaires: a meta-analysis. Health Qual Life Outcomes. 2010;8(1):99.

13. Pasma A, van't Spijker A, Hazes JM, Busschbach JJ, Luime JJ. Factors associated with adherence to pharmaceutical treatment for rheumatoid arthritis patients: a systematic review. Semin Arthritis Rheum. 2013;43(1):18-28.

14. Curtis JR, Bykerk VP, Aassi M, Schiff M. Adherence and persistence with methotrexate in rheumatoid arthritis: a systematic review. J Rheumatol. 2016;43(11):1997-2009.

15. Costedoat-Chalumeau N, Pouchot J, Guettrot-Imbert G, et al. Adherence to treatment in systemic lupus erythematosus patients. Best Pract Res Clin Rheumatol. 2013;27(3):329-340.

16. Nguyen TM, La Caze A, Cottrell N. What are validated self-report adherence scales really measuring?: a systematic review. Br J Clin Pharmacol. 2014;77(3):427-445.

17. de Klerk E, van der Heijde D, van der Tempel H, van der Linden S Development of a questionnaire to investigate patient compliance with antirheumatic drug therapy. J Rheumatol. 1999;26(12):2635-2641.

18. Lam WY, Fresco P. Medication adherence measures: an overview. Biomed Res Int. 2015;2015:217047.

19. Smolen JS, Landewé R, Breedveld FC, et al. EULAR recommendations for the management of rheumatoid arthritis with synthetic and biological disease-modifying antirheumatic drugs: 2013 update. Ann Rheum Dis. 2014;73(3):492-509.

20. Gossec L, Smolen JS, Ramiro S, et al. European League Against Rheumatism (EULAR) recommendations for the management of psoriatic arthritis with pharmacological therapies: 2015 update. Ann Rheum Dis. 2016;75(3):499-510.

21. Smolen JS, Braun J, Dougados M, et al. Treating spondyloarthritis, including ankylosing spondylitis and psoriatic arthritis, to target: recommendations of an international task force. Ann Rheum Dis. 2014; 73(1):6-16.

22. Bertsias G, Ioannidis JP, Boletis J, et al. EULAR recommendations for the management of systemic lupus erythematosus. Report of a Task Force of the EULAR Standing Committee for International Clinical Studies Including Therapeutics. Ann Rheum Dis. 2008;67(2):195-205.
23. Horne R, Weinman J, Hankins M. The beliefs about medicines questionnaire: the development and evaluation of a new method for assessing the cognitive representation of medication. Psychol Health. 1999;14(1):1-24.

24. Chu LH, Kawatkar AA, Gabriel SE. Medication adherence and attrition to biologic treatment in rheumatoid arthritis patients. Clin Ther. 2015;37(3):660.e8-666.e8.

25. Bonafede M, Johnson BH, Tang DH, Shah N, Harrison DJ, Collier DH. Etanercept-methotrexate combination therapy initiators have greater adherence and persistence than triple therapy initiators with rheumatoid arthritis. Arthritis Care Res (Hoboken). 2015;67(12):1656-1663.

26. Kim G, Barner JC, Rascati K, Richards K. Examining time to initiation of biologic disease-modifying antirheumatic drugs and medication adherence and persistence among texas medicaid recipients with rheumatoid arthritis. Clin Ther. 2016;38(3):646-654.

27. Kumar K, Raza K, Nightingale P, et al. Determinants of adherence to disease modifying anti-rheumatic drugs in White British and South Asian patients with rheumatoid arthritis: a cross sectional study. $B M C$ Musculoskelet Disord. 2015;16(1):396.

28. Gadallah MA, Boulos DN, Dewedar S, Gebrel A, Morisky DE. Assessment of rheumatoid arthritis patients' adherence to treatment. Am J Med Sci. 2015;349(2):151-156

29. De Cuyper E, De Gucht V, Maes S, Van Camp Y, De Clerck LS. Determinants of methotrexate adherence in rheumatoid arthritis patients. Clin Rheumatol. 2016;35(5):1335-1339.

30. Salaffi F, Carotti M, Di Carlo M, Farah S, Gutierrez M. Adherence to anti-tumor necrosis factor therapy administered subcutaneously and associated factors in patients with rheumatoid arthritis. JCR J Clin Rheumatol. 2015;21(8):419-425.

31. Hromadkova L, Soukup T, Vlcek J. Quality of life and drug compliance: their interrelationship in rheumatic patients. J Eval Clin Pract. 2015;21(5):919-924.

32. Abdul-Sattar AB, Abou El Magd SA. Determinants of medication non-adherence in Egyptian patients with systemic lupus erythematosus: Sharkia Governorate. Rheumatol Int. 2015;35(6):1045-1051.

33. Betegnie AL, Gauchet A, Lehmann A, et al. Why do patients with chronic inflammatory rheumatic diseases discontinue their biologics? an assessment of patients' adherence using a self-report questionnaire. J Rheumatol. 2016;43(4):724-730.

34. Lyu R, Govoni M, Ding Q, et al. Treatment persistence among patients with rheumatoid disease (RA, AS, PsA) treated with subcutaneous biologics in Germany. Rheumatol Int. 2016;36(1):143-153.

35. Machado MA, Moura CS, Ferré F, Bernatsky S, Rahme E, Acurcio F de A. Treatment persistence in patients with rheumatoid arthritis and ankylosing spondylitis. Rev Saude Publica. 2016;50:50.

36. Glintborg B, Højgaard P, Lund Hetland M, et al. Impact of tobacco smoking on response to tumour necrosis factor-alpha inhibitor treatment in patients with ankylosing spondylitis: results from the Danish nationwide DANBIO registry. Rheumatology (Oxford). 2016;55(4): 659-668.

37. Højgaard P, Glintborg B, Hetland ML, et al. Association between tobacco smoking and response to tumour necrosis factor $\alpha$ inhibitor treatment in psoriatic arthritis: results from the DANBIO registry. Ann Rheum Dis. 2015;74(12):2130-2136.

38. Morgan C, McBeth J, Cordingley L, et al. The influence of behavioral and psychological factors on medication adherence over time in rheumatoid arthritis patients: a study in the biologics era. Rheumatology (Oxford). 2015;54(10):1780-1791.

39. DiMatteo MR, Giordani PJ, Lepper HS, Croghan TW. Patient adherence and medical treatment outcomes: a meta-analysis. Med Care. 2002;40(9):794-811.

40. Souto A, Maneiro JR, Gómez-Reino JJ. Rate of discontinuation and drug survival of biologic therapies in rheumatoid arthritis: a systematic review and meta-analysis of drug registries and health care databases. Rheumatology (Oxford). 2016;55(3):523-534.

41. Brown MT, Bussell JK. Medication adherence: WHO cares? Mayo Clin Proc. 2011;86(4):304-314. 
42. Söderlin MK, Petersson IF, Geborek P. The effect of smoking on response and drug survival in rheumatoid arthritis patients treated with their first anti-TNF drug. Scand J Rheumatol. 2012; 41(1):1-9.

43. Fagerli KM, Lie E, van der Heijde D, et al. The role of methotrexate co-medication in TNF-inhibitor treatment in patients with psoriatic arthritis: results from 440 patients included in the NOR-DMARD study. Ann Rheum Dis. 2014;73(1):132-137.

44. Michetti P, Weinman J, Mrowietz U, et al. Impact of treatment-related beliefs on medication adherence in immune-mediated inflammatory diseases: results of the Global ALIGN Study. Adv Ther. 2017;34(1):91-108.

45. Nota I, Drossaert CH, Taal E, van de Laar MA. Patients' considerations in the decision-making process of initiating disease-modifying antirheumatic drugs. Arthritis Care Res (Hoboken). 2015;67(7):956-964.

46. Martin RW, Head AJ, René J, et al. Patient decision-making related to antirheumatic drugs in rheumatoid arthritis: the importance of patient trust of physician. J Rheumatol. 2008;35(4):618-624.

47. Payne RA. The epidemiology of polypharmacy. Clin Med (Lond). 2016; 16(5):465-469.

48. Chapman RH, Benner JS, Petrilla AA, et al. Predictors of adherence with antihypertensive and lipid-lowering therapy. Arch Intern Med. 2005;165(10):1147-1152.

49. Tavares NU, Bertoldi AD, Mengue SS, et al. Fatores associados à baixa adesão ao tratamento farmacológico de doenças crônicas no Brasil [Factors associated with low adherence to medicine treatment for chronic diseases in Brazil]. Rev Saude Publica. 2016;50(Suppl 2):10s. Portuguese.

50. de Klerk E, van der Heijde D, Landewé R, van der Tempel H, Urquhart J, van der Linden S. Patient compliance in rheumatoid arthritis, polymyalgia rheumatica, and gout. J Rheumatol. 2003;30(1):44-54.
51. Maneiro JR, Salgado E, Gomez-Reino JJ. Immunogenicity of monoclonal antibodies against tumor necrosis factor used in chronic immunemediated inflammatory conditions: systematic review and meta-analysis. JAMA Intern Med. 2013;173(15):1416-1428.

52. Garcia-Gonzalez A, Richardson M, Garcia Popa-Lisseanu M, et al. Treatment adherence in patients with rheumatoid arthritis and systemic lupus erythematosus. Clin Rheumatol. 2008;27(7):883-889.

53. Pasma A, Schenk CV, Timman R, et al. Non-adherence to diseasemodifying antirheumatic drugs is associated with higher disease activity in early arthritis patients in the first year of the disease. Arthritis Res Ther. 2015;17(1):281.

54. Clifford S, Barber N, Horne R. Understanding different beliefs held by adherers, unintentional nonadherers, and intentional nonadherers: application of the Necessity-Concerns Framework. J Psychosom Res. 2008;64(1):41-46.

55. Foot H, La Caze A, Gujral G, Cottrell N. The necessity-concerns framework predicts adherence to medication in multiple illness conditions: a meta-analysis. Patient Educ Couns. 2016;99(5):706-717.

56. Neame R, Hammond A. Beliefs about medications: a questionnaire survey of people with rheumatoid arthritis. Rheumatology (Oxford). 2005;44(6): $762-767$.

57. de Vries ST, Keers JC, Visser R, et al. Medication beliefs, treatment complexity, and non-adherence to different drug classes in patients with type 2 diabetes. J Psychosom Res. 2014;76(2):134-138.

58. Larkin AT, Hoffman C, Stevens A, Douglas A, Bloomgarden Z. Determinants of adherence to diabetes treatment. $J$ Diabetes. 2015;7(6):864-871.

59. Andersson Sundell K, Jönsson AK. Beliefs about medicines are strongly associated with medicine-use patterns among the general population. Int J Clin Pract. 2016;70(3):277-285.
Patient Preference and Adherence

\section{Publish your work in this journal}

Patient Preference and Adherence is an international, peer-reviewed, open access journal that focuses on the growing importance of patient preference and adherence throughout the therapeutic continuum. Patient satisfaction, acceptability, quality of life, compliance, persistence and their role in developing new therapeutic modalities and compounds to optimize

\section{Dovepress}

clinical outcomes for existing disease states are major areas of interest for the journal. This journal has been accepted for indexing on PubMed Central. The manuscript management system is completely online and includes a very quick and fair peer-review system, which is all easy to use. Visit http://www. dovepress.com/testimonials.php to read real quotes from published authors. 